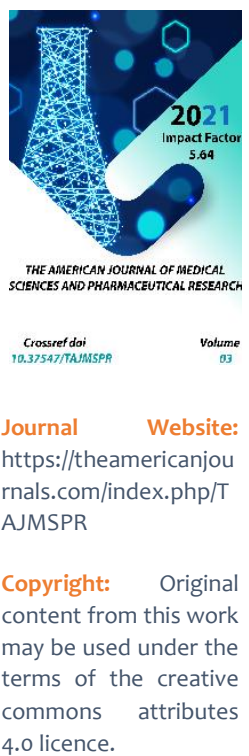

\section{Clinical Application Of Dental Photography By A Dentist}

\author{
Xolboeva Nasiba Asrorovna \\ Assistant, Samarkand State Medical Institute, Department Of Therapeutic Dentistry, Uzbekistan \\ Turaev Alim Baxriddinovich \\ Assistant, Samarkand State Medical Institute, Department Of Therapeutic Dentistry, Uzbekistan \\ Islamova Nilufar Bustanovna \\ Assistant, Samarkand State Medical Institute, Department Of Therapeutic Dentistry, Uzbekistan \\ Davronov Sodiqjon Valijon O'g'li \\ Doctor, Samarkand State Medical Institute, Department Of Therapeutic Dentistry, Uzbekistan \\ Turaeva Kamila Furqat Qizi \\ Doctor, Samarkand State Medical Institute, Department Of Therapeutic Dentistry, Uzbekistan
}

\title{
ABSTRACT
}

"One picture is worth a thousand words" - Frederic Bernard Currently, many dentists use a camera in their daily practice, turning into photographers for a while. In aesthetic dentistry, the main tool for diagnosis and communication is the art and practice of obtaining a photo. The use of dental photography is the standard of modern dental treatment, since photographs of the results of treatment facilitate understanding of the features of dental intervention. And most importantly, visualization increases the motivation of the patient and promotes the establishment of friendly relations between him and the doctor.

\section{KEYWORDS}

Dental photography, smile, patient motivation.

\section{INTRODUCTION}

Today, an increasing number of patients seek dental care not only for the purpose of recreating the function of chewing or speech, but also for aesthetic purposes. In addition, the dental literacy of people and, accordingly, the requirements for the quality of treatment are increasing. This requires new tools, visibility, motivation, which is what digital photography is. In the photo, the patient can directly see the existing problems with the teeth and dentition, the mucous membrane and understand the need for complete treatment.Photos of the patient's smile and oral cavity are the most important diagnostic criteria for treatment planning; provide an opportunity for remote communication between colleagues-dentists of various specialties, the possibility of remote consultations of patients and presentations of 
possible treatment options. The patient's motivation for treatment increases significantly, since he sees the same thing as the doctor, and perceives information about the state of his health by visual confirmation of the diagnoses made. Without digital photography, high-quality aesthetic rehabilitation of the patient is impossible, since during direct restoration, the dentist-therapist focuses only on the problem tooth, and for successful treatment today it is necessary to understand the image of the patient as a whole and its perception by surrounding people and themselves remotely, which can only give an image in a photo. Almost any consultation of a good dentist today begins with a series of photos: portrait and inside the mouth. Portrait photos allow you to evaluate the shape of a smile, the position of the lips, the architectonics of the face for aesthetic restoration of the anterior group. Thus, today, dental digital photography is the most important tool for motivating patients to treatment and communication between doctors of different profiles.

Photos before and after treatment, as well as at its stages, contribute to monitoring the quality level of the doctor's work, allow you to create a patient's portfolio and help in resolving controversial legal issues, if they arise.

To study the influence of dental digital photography on the level of motivation of patients for dental treatment and to assess the quality of direct restoration after treatment.

\section{MATERIALS AND METHODS}

The study was conducted in the city dental clinic of the city of Samarkand, which was attended by 30 people aged 16 to 45 years. The main complaints of patients when seeking dental care were: aesthetic dissatisfaction of the anterior group of teeth (cosmetic defect of restorations), pain from thermal stimuli.

The following diagnoses were made: K02. 1 dentin caries (72\% of cases), K04. o pulpitis (45\%), Ko 4.5 chronic apical periodontitis (24\%), K03.1 wedge-shaped defect (37\%), K03.2 enamel erosion (25\%), K03.0 increased tooth eras ability (15\%), K07.3 anomalies of the position of individual teeth (28\%), K02.8 defects of hard tooth tissues (57\%). S02. 5 tooth injury (10\%), K05.1 chronic gingivitis (28\%), anomalies of the shape of the dental arches (34\%), Each patient was diagnosed with several diagnoses (the percentage was considered from the total number of examined patients).

All patients were divided into two groups: the control group included 15 people who were treated using the full photo protocol, and 20 patients from the other group were treated according to the traditional scheme. Before the therapeutic treatment, according to the indications, professional oral hygiene was carried out. The evaluation of the results of treatment with direct restorations was carried out according to the following parameters: the appearance of the patient with restoration (in the anterior part of the upper and lower jaw), a filling defect, lack of marginal fit, excess material at the filling-tooth border, roughness of the filling, gloss, compliance with the color of adjacent natural teeth, compliance with aesthetic parameters and functional norms, occlusive contacts with antagonist teeth and proximal contacts, the severity of morphological parameters of the tooth. We also evaluated the percentage of oral sanitation in the group of patients who passed the photo protocol, and in the group of patients without digital photography. The 
study was conducted for 10 months, after the treatment, patients were twice invited for repeated examinations.

\section{RESULTS AND DISCUSSIONS}

In the course of the study, the following results were obtained: in the control group of the examined, the level of motivation for dental treatment was very high (100\%) and all 15 people had a complete rehabilitation of the oral cavity, including consultations and treatment with other dental specialists. $70 \%$ of patients in this group underwent direct restoration. Treatment of pathology of hard tissues of teeth using aesthetic restoration is very relevant today. In another group of patients, the level of motivation for dental treatment was much lower. Out of 15 people, only 4 people (38\%) showed up for repeated admission and treatment, the remaining 11 (62\%) were limited to treating one tooth that caused pain.

\section{CONCLUSION}

Digital photography greatly facilitates the exchange of data and informing patients. Photos in standard projections greatly facilitated aesthetic analysis, revealing an assessment of the enamel distribution, optical effects, hypoplasia areas and the transparency of the cutting edge. Clinical images made it possible to accurately analyze the appearance of the smile and dentition. A smile is a natural frame of the dentition. Photos of smiles of varying degrees of severity gave us important information for planning restoration treatment. In our opinion, the final diagnosis should be made only after analyzing the full series of images before treatment. Correct diagnosis is the main condition for successful restoration treatment. The use of the photo protocol in the control group increased the motivation for total dental treatment, compared to the usual traditional treatment and explanation simply in the words of a doctor. In such a technologically complex profession as dentistry, it is necessary to objectively approach the choice of a camera.

\section{REFERENCE}

1. Kravets TP. Documents on the history of the invention of photography. No $360,361,380,388-389$.

2. Laws R. The author's guide to controlling the photograph. No 213-218

3. Douglas Terry, Willie Geller. Aesthetic and restorative dentistry.

4. Domenico Massironi. Precision and Aesthetics.

5. Yordi Manauta, Anna Salat. Layers. Atlas of layered composite restorations.

6. Wander P. Gordon P. Specific applications of dental photography.

7. A NEW STAGE OF REFORMING THE TRAINING OF LEGAL PERSONNEL. F Mukhitdinova Review of law sciences 2 (1), 2017

8. Ideas and teachings on the concept of spiritual and moral education of the younger generation in Uzbekistan FA Mukhitdinova, SS Agzamkhodzhayeva Eurasian Union of Scientists, 2019.

9. Problems of power and management in the works of Eastern thinkers FA Mukhitdinova, HM Mukhammedov High School, 35-38, 2015.

10. Implementation of human rights protection in Uzbekistan IV 
The American Journal of Medical Sciences and Pharmaceutical Research

(ISSN - 2689-1026)

Published: September 17, 2021 | Pages: 10-13

IMPACT FACTOR

2021: 5.64

Doi: https://doi.org/10.37547/TAJMSPR/Volume03Issue09-03

OCLC - 1121105510

Kudryavtsev Eurasian Union of

Scientists, 2019.

11. The origins of the idea of civil society

FA Mukhitdinova, IN Kudryavtsev

ACTUAL ASPECTS OF MODERN

SCIENCE, 102-109, 2017. 